\title{
Diffusion-Limited Aggregation with Active Edge Diffusion
}

\author{
Holger Röder, Karsten Bromann, Harald Brune, and Klaus Kern \\ Institut de Physique Expérimentale, EPF Lausanne, CH-1015 Lausanne, Switzerland \\ (Received 2 December 1994)
}

\begin{abstract}
Diffusion-limited aggregation of $\mathrm{Ag}$ on $\mathrm{Pt}(111)$ and $\mathrm{Ag}(111)$ has been studied by scanning tunneling microscopy. In both metal-on-metal systems fractal growth shapes are found in an extended range of deposition temperature and deposition flux. In contrast to classic hit-and-stick diffusion-limited aggregation simulations the average branch width of the ramified aggregates is not monatomic and increases with increasing deposition temperature, which is attributed to an active edge diffusion. From a quantitative analysis of the exponential variation of the branch width with temperature we determine the diffusion constants for edge diffusion.
\end{abstract}

PACS numbers: 68.55.Jk, 61.16.Ch, 61.43.Hv, 68.70.+w

A rich variety of dendritic and randomly ramified (fractal) structures are observed in physical, chemical, or biological systems [1]. They often result from aggregation under highly nonequilibrium conditions with material transport via diffusion being the growth-determining process. The models used to describe these processes have aimed at reproducing the growth patterns following essentially two approaches: the surface tension approach of dendritic growth [2] and the diffusion-limited aggregation (DLA) model $[3,4]$. In the first approach, dendritic patterns appear as a result of competition between the nonlocal diffusion field, which amplifies the growth at aggregate sites having the steepest gradient of supply-atom concentration, and the surface tension, favoring flat interfaces. In the DLA computer model clusters are formed as particles diffusing randomly on a $2 \mathrm{D}$ lattice stick irreversibly to the perimeter of the growing aggregate. DLA clusters are quite different from dendrites; they are irregular, randomly branched aggregates with no preferred axes. Despite a considerable theoretical effort $[5,6]$, the relationship between ordered interfacial patterns and fractal DLA aggregates is not well established. In a recent experimental study of diffusion-limited $\mathrm{Ag}$ aggregation on a $\operatorname{Pt}(111)$ surface at low temperatures, we have demonstrated that the link between the two approaches is the finite edge diffusion at the perimeter of the aggregate [7].

The most stringent condition of classic DLA simulations is that once an adatom is attached to the growing cluster, it is trapped immediately with no further diffusion. For the growth of 2D metal clusters, however, this does not hold in general, even at low temperatures. For $\mathrm{Ag}$ aggregation on $\mathrm{Pt}(111)$, edge diffusion was found to be active down to at least $60 \mathrm{~K}$ [7]. On fcc (111) surfaces, edge diffusion barriers are often found to be orientation dependent (there are two types of close-packed steps with microscopically different structure) [7,8]. The anisotropy in the speed of migration along the two edges might give rise to anisotropic growth patterns. Such dendritic patterns are indeed formed by $\mathrm{Ag}$ on $\mathrm{Pt}(111)$ in the temperature range $110-130 \mathrm{~K}$ for high growth speeds. A transition to randomly ramified fractal growth is observed upon lowering the deposition flux [7]. In general, if edge diffusion is anisotropic, dendrites result if the growth rate is higher than that of the slower edge diffusion. With increasing substrate temperature, the exponentially increasing rate of edge diffusion rapidly dominates the growth speed, and randomly ramified fractals result.

In the present Letter we report on the first detailed experimental study of diffusion-limited aggregation with active edge diffusion in the noise dominated random growth regime. For Ag aggregation on the (111) surfaces of $\mathrm{Pt}$ and Ag, randomly ramified islands are found to grow in extended temperature regimes. The characteristic parameter describing the growth of these fat fractals is the average branch width, which is found to increase exponentially with deposition temperature. From a quantitative analysis of the temperature dependence of the branch width, we determine edge diffusion constants.

The experiments were performed with a variabletemperature scanning tunneling microscope (STM) mounted in UHV, which operates in the temperature range 25 to $800 \mathrm{~K}[9,10]$. The $\mathrm{Pt}(111)$ crystal has been cleaned by repeated cycles of $\mathrm{Ar}$ ion bombardment $(750 \mathrm{eV})$ at $800 \mathrm{~K}$ and subsequent annealing at $1200 \mathrm{~K}$. The $\operatorname{Ag}(111)$ surface was prepared by epitaxial growth of a 40 monolayer (ML) thick Ag film on the $\mathrm{Pt}(111)$ surface [11]. The Ag aggregates were grown by vapor-phase epitaxy with a molecular beam epitaxy Knudsen cell at a background pressure better than $2 \times 10^{-10}$ mbar. The STM images were measured in constant current mode with tunneling currents $I=1.0$ to $3.0 \mathrm{nA}$ at sample voltages $U=-0.5$ to $-1.5 \mathrm{~V}$. All images shown here were recorded in differential mode, which means that the derivatives of the lines of constant tunnel current were recorded.

In Fig. 1 we show a series of STM images characterizing fractal $\mathrm{Ag}$ aggregates on $\mathrm{Pt}(111)$ grown at the temperatures indicated. The substantial increase of the average branch width with increasing temperature is obvious. At the lowest temperature, $110 \mathrm{~K}$, large clusters $(\sim 3000 \mathrm{Ag}$ 
$\mathrm{T}=110 \mathrm{~K}$

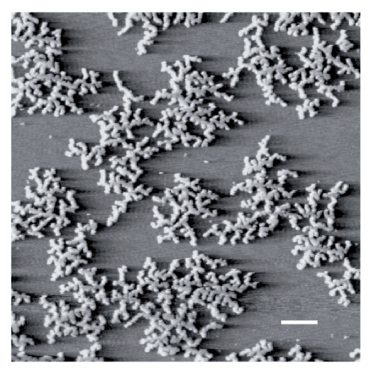

(a)

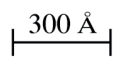

$\mathrm{T}=220 \mathrm{~K}$

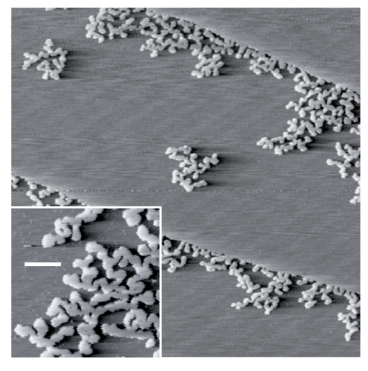

(c)
$500 \AA$

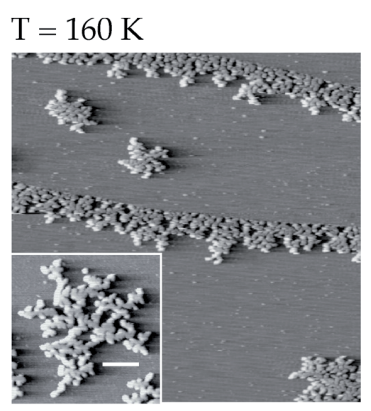

(b)

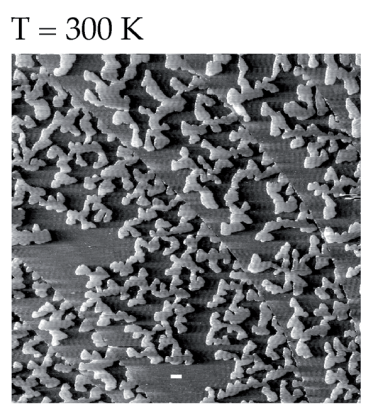

(d)
$1500 \AA$
FIG. 1. Fractal Ag aggregation on $\mathrm{Pt}(111)$ as a function of deposition temperature. The $\mathrm{Ag}$ coverage is in (a) - (c) $\Theta=0.1 \mathrm{ML}$ and in (d) $\Theta=0.4 \mathrm{ML}$, the deposition flux is in (a) $R=7 \times 10^{-5} \mathrm{ML} / \mathrm{s}$ and in (b) - (d) $R=1.1 \times 10^{-3} \mathrm{ML} / \mathrm{s}$. The STM images in (a) and the insets in (b) and (c) have the same lateral scale (the white ruler measures $100 \AA$ ) revealing the branch width broadening with increasing temperature.

atoms) with an open ramified structure aggregate on the Pt(111) terraces. The branches of the clusters show no preferred orientation, i.e., show no simple correlation with the symmetry axes of the substrate. The branch thickness ( $w=2.5 \pm 1$ atoms) is nearly constant over the entire aggregate and is much smaller than its radius of gyration. The appearance of the Ag aggregates at $110 \mathrm{~K}$ is very similar to that of fractal aggregates simulated with the DLA computer codes. A mass-length scaling analysis does, indeed, determine the expected fractal DLA dimension [7]. At $300 \mathrm{~K}$, the other extreme in the temperature series, no homogeneous nucleation occurs on terraces as wide as $2000 \AA$. The mean free path $\Lambda_{D}$ of a diffusing $\mathrm{Ag}$ adatom at this temperature is much larger than the average step separation and $\mathrm{Ag}$ grows from the ascending steps. Despite the step nucleation, the randomly ramified growth shape of the Ag deposit remains clearly recognizable even at the total coverage of $\Theta=0.4 \mathrm{ML}$. The average branch width measured in Fig. 1(d) is $w=75 \pm 10$ atoms. At a coverage of $\Theta=0.1 \mathrm{ML}$, a factor of 4 lower than the previous coverage, the average branch width is still found to be $w=37 \pm 6$ atoms [12], i.e., has decreased only by a factor of 2. This nonlinear behavior of $w(\Theta)$ can be re- lated to the screening effect of fractal growth [4]. In addition, this also indicates that at higher temperatures, significant edge diffusion occurs, and the aggregate perimeter is partially screened by the growing tips. In the intermediate temperature range $\mathrm{Ag}$ aggregates of fractal shape are seen to nucleate simultaneously on terraces and at ascending steps. It is important to note that the branch width of terrace islands and step fingers are found to be identical within the error margin. At temperatures of 160 and $220 \mathrm{~K}$, at a total coverage of $0.1 \mathrm{ML}$, we measure $w=8 \pm 2$ and $16 \pm 3$ atoms, respectively.

The aggregation behavior of $\mathrm{Ag}$ on the $\mathrm{Ag}(111)$ surface is very similar. Fractal island shapes are found in the temperature range between 80 and $160 \mathrm{~K}$, with the branch width increasing exponentially with deposition temperature. In Fig. 2 we show an example of ramified $\mathrm{Ag}$ islands grown at $150 \mathrm{~K}$. The average sized cluster contains about $1500 \mathrm{Ag}$ atoms and its branch width is measured to be $w=18 \pm 4$ atoms. The decreased temperature range of fractal island shapes of $\mathrm{Ag} / \mathrm{Ag}(111)$ with respect to $\mathrm{Ag} / \mathrm{Pt}(111)$ is related to the lower edge diffusion barrier (see below) and the lower kink energy of the homoepitaxial system. Both factors favor the growth of compact island shapes at higher temperatures, which are energetically more favorable than fractals due to their minimized island contour energy.

The growth of a fractal island is ultimately related to the simple geometric effect that a small tip on an island tends to become longer because incoming adatoms attach preferentially to that tip [4]. In classic hit-and-stick DLA simulations this screening effect is active from the very beginning of the deposition process. In DLA with active edge diffusion, however, tips develop only above a critical island size. For aggregation of $\mathrm{Ag}$ on $\mathrm{Pt}(111)$ between 60 and $110 \mathrm{~K}$, this critical size has been found to be the heptamer [7,13]. Up to this size Ag clusters form compact shapes; branching is observed only for larger clusters. It

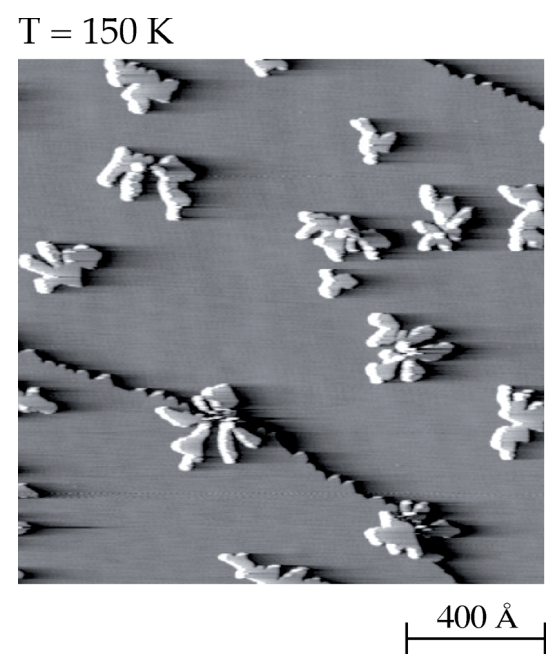

FIG. 2 Fractal $\mathrm{Ag}$ aggregates on $\mathrm{Ag}(111)$ grown at $150 \mathrm{~K}$ $\left(\Theta=0.1 \mathrm{ML}, R=1.1 \times 10^{-3} \mathrm{ML} / \mathrm{s}\right)$. 
was suggested by Pimpinelli, Villain, and Wolf [14] that this critical size for island branching is mainly controlled by the diffusion constant $D_{e}$ for edge atoms.

Mobile atoms adsorbed at the island perimeter can migrate along the step edge and either attach to an existing kink site or meet a second mobile edge atom nucleating new kinks. The relative probability of these events depends on the lateral size $L$ of the step edge and the impinging two-dimensional adatom flux $I$ on the island. If the average time $\tau_{e}$ for a mobile edge atom to "scan" the entire step length $L$ is smaller than the average time $\tau_{i}=1 / I$ between two successive impinging events, the island will grow in a compact shape. A shape instability, i.e., the branching of the compact seed cluster, occurs if nucleation events dominate the slow edge diffusion and the step grows perpendicularly. Therefore, the critical edge length $L_{c}$ beyond which the island growth is unstable with respect to branching is given by the condition $\tau_{e}=\tau_{i}$. Using the Einstein relation $L^{2}=D_{e} \tau_{e}\left[D_{e}=\frac{1}{2} \nu_{e} \exp \left(-E_{e} / k T\right)\right.$ in atomic units $]$, we obtain the following criterion for the fractal shape instability: $L_{c} \approx\left(D_{e} / I\right)^{1 / 2}$. On a hexagonal lattice, to a good approximation, the critical island size equals the average branch width $L_{c}=w$ (see Fig. 3 in Ref. [7]). The instability criterion was originally proposed by Pimpinelli et al. in somewhat modified form [15] and has been supported recently by Monte Carlo simulations performed by Bartelt and Evans [16].

The average branch width $w(T)$ has been evaluated from the STM images by measuring the total branch length and dividing by the number of atoms deposited on the corresponding area. For small widths, the STM tip is found to image the branches about $6 \AA$ larger than their actual size, which is consistent with the usual "smearing" observed at monatomic steps of closepacked metal surfaces [17]. The two-dimensional flux $I$, which corresponds to the number of atoms arriving at the perimeter of an aggregate, has been evaluated as follows: every island has an "active" area $\Lambda_{D}^{2}\left(\Lambda_{D}\right.$

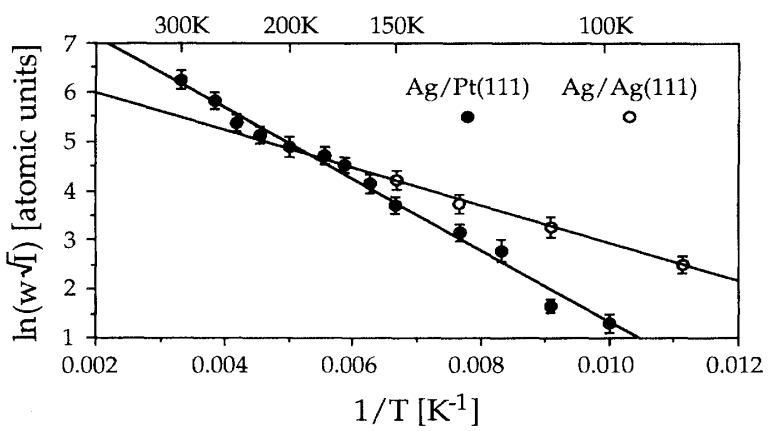

FIG. 3. Arrhenius behavior of the average branch width $w$ (weighted with $\sqrt{I}$, with $I$ being the impinging two-dimensional adatom flux) of $\mathrm{Ag}$ fractals grown on $\mathrm{Pt}(111)(\bullet)$ and on $\operatorname{Ag}(111)(\circ)$, respectively. All data have been evaluated at $\Theta=0.1 \mathrm{ML}$ and $R=1.1 \times 10^{-3} \mathrm{ML} / \mathrm{s}$. being the average migration distance of an adatom at a given temperature) in which all arriving atoms will on average perform their random walk towards the island. This "active" area is given by the inverse saturation island density, $\Lambda_{D}^{2}=1 / n_{x}$. The impinging flux onto the island perimeter is therefore $I=R / n_{x}$, with $R$ being the deposition flux onto the substrate. Here we assume that the sticking coefficient at the aggregate perimeter is 1 , and that sticking is irreversible. For metal-on-metal systems this is a good approximation at low temperatures, but breaks down at higher temperatures where the twodimensional spreading pressure of the admetal becomes non-negligible. For $\mathrm{Ag}$ on $\mathrm{Pt}(111)$, this transition from kinetic to thermodynamic behavior has been determined to occur above $350 \mathrm{~K}$ [9], i.e., well above the data range for the analysis of the fractal island shapes.

Since above $150 \mathrm{~K}$ most islands nucleate at preexisting substrate steps, no $n_{x}$ data are available for the temperature range $150-300 \mathrm{~K}$. The flux $I$ has therefore been evaluated by the introduction of an active area of a substrate step, defined as the product $\Lambda_{\text {cri }} \Lambda_{\text {emp }}$. Here, $\Lambda_{\text {cri }}$ is the largest substrate terrace width with no homogeneous nucleation, and $\Lambda_{\mathrm{emp}}$ is a fit parameter, which was determined to be $300 \AA$ in order to achieve correspondence with an extrapolation of the values obtained in the terrace nucleation regime. Since a finger growing from a step collects about twice as many atoms per unit time than an island tip, it follows that $I \approx 2 R\left[\Lambda_{\text {cri }}(300 \AA)\right]^{-1}$. At $130 \mathrm{~K}$ this $I$ value matches the one obtained by $I=R / n_{x}$ within $10 \%$.

In Fig. 3 we show the temperature variation of the measured $w \sqrt{I} \approx \sqrt{D_{e}}$ values for $\mathrm{Ag}$ aggregation on $\operatorname{Pt}(111)$ and $\mathrm{Ag}(111)$. Both data sets lie on a straight line in the Arrhenius representation. This behavior allows one to determine rather precisely the edge diffusion constants. From the slope of the line that fits the STM data, surprisingly low edge diffusion barriers $E_{e}$ are calculated: $125 \pm 10 \mathrm{meV}$ for $\mathrm{Ag} / \mathrm{Pt}(111)$ and $65 \pm 10 \mathrm{meV}$ for $\mathrm{Ag} / \mathrm{Ag}(111)$. The relative error is somewhat larger for the homoepitaxial system due to the smaller accessible temperature range. These barriers are "average" values as in the fractal growth regime both microscopically different steps contribute to branching. In the heteroepitaxial as well as in the homoepitaxial system, the determined step edge diffusion barriers are comparable with the terrace diffusion barriers $E_{D}$ of $157 \mathrm{meV}$ [Ag/Pt(111)] [13] and $100 \mathrm{meV} \mathrm{[Ag/Ag(111)]} \mathrm{[18].}$

It has usually been argued in the literature that the requirement for fractal island growth is $E_{D} \ll E_{e}[8,19]$, a condition which appears to be violated for the present metal-on-metal systems. This criterion, however, is valid only if the attempt frequencies of terrace and edge diffusion are comparable, i.e., $\nu_{D} \approx \nu_{e}$, which cannot be assumed a priori. Our data indicate indeed that the $\nu_{e}$ values are significantly lower than the preexponential factors for terrace diffusion $\left[\nu_{D} \approx 10^{13} \mathrm{~Hz}\right.$ for $\mathrm{Ag} / \mathrm{Pt}(111)$ [13]]. Using the empirical formula $L_{c}=w=1.7\left(D_{e} / I\right)^{1 / 2}$ from 
the Monte Carlo simulations of Bartelt and Evans [16] we can conclude that the proportionality constant is of the order of 1 (in atomic units). Thus, from the intersection of the two data sets with the ordinate the step edge preexponetial factors $\nu_{e}$ of $1 \times 10^{-8 \pm 1}$ and $1 \times 10^{-6 \pm 1} \mathrm{~Hz}$ are determined. These low diffusion prefactors are indicative for an exchange type of diffusion involving concerted motions [20,21]. Thus, the fractal growth of Ag aggregates on the (111) surfaces of $\mathrm{Pt}$ and $\mathrm{Ag}$ is to first order triggered by a slow step edge diffusion mechanism associated with a low diffusion prefactor of the order $D_{0} \approx$ $4 \times 10^{-8 \pm 2} \mathrm{~cm}^{2} / \mathrm{s}$. The correct criterion for fractal aggregation in epitaxial metal growth (with typical deposition rates) reads $D_{d} \gg D_{e}$. Thus, a large difference between the lateral and step edge diffusion constants is required, and is fulfilled in the present case due to the large difference in the preexponential factors of step and terrace diffusion (probably concerted exchange versus single hopping diffusion).

We want to comment finally on the growth of classic hit-and-stick DLA fractals in fcc (111) metal epitaxy. DLA fractals imply completely frozen edge diffusion and a monatomic branch width $w=1$. From extrapolation of the $\mathrm{Ag} / \mathrm{Pt}(111)$ data in Fig. 3, we would expect to grow monatomic DLA aggregates at temperatures below about $85 \mathrm{~K}$. However, also at temperatures as low as $60-80 \mathrm{~K}$ the $\mathrm{Ag}$ aggregates form compact clusters up to the heptamer before branching is observed and the branch width never becomes monatomic but stays diatomic (see Figs. 3 in Refs. [7,13]). This incapacity of classic DLA clusters to aggregate on fec (111) metal surfaces is related to local distortions of the adsorption potential around the cluster edges [22,23]. This local potential modification not only generates an "empty" zone of adatoms surrounding a cluster but also guides the adatoms approaching the cluster edge towards certain adsorption sites. In the field-ion microscopy studies of Ir attachment to Ir clusters on $\operatorname{Ir}(111)$ all adatoms have been found to attach exclusively to twofold coordinated edge sites omitting single coordination (see the "attachment" maps, Figs. 2 and 3 in Refs. [22] and [23], respectively). Because of selective sticking, branching is suppressed for small clusters $(n<7)$ and the branch width is limited to $w=2$, the smallest width at which all atoms are coordinated at least twofold.

In conclusion, we have studied experimentally the influence of an active edge diffusion on the DLA of metal atoms on fcc (111) metal surfaces. The resulting fat fractals are characterized by their branch width $w$, which is found to increase exponentially with growth temperature. From a quantitative analysis of the temperature dependence we have determined edge diffusion constants. We have further provided evidence that classic hit-and-stick DLA aggregates with monatomic width are unlikely to be formed on fcc (111) surfaces due to local potential modifications at the cluster edges. Our study clearly demonstrates the limitations of classic DLA in the simulation of the aggregation kinetics in metal epitaxy. Even a qualitative kinetic modeling requires the detailed understanding of the microscopic diffusion and sticking processes.

This work has been supported by the Schweizerischer Nationalfonds.

[1] T. Vicsek, Fractal Growth Phenomena (World Scientific, Singapore, 1989).

[2] J. S. Langer, Rev. Mod. Phys. 52, I (1980).

[3] T. A. Witten and L. M. Sander, Phys. Rev. Lett. 47, 1400 (1981).

[4] P. Meakin, in Phase Transitions and Critical Phenomena, edited by C. Domb and J. L. Lebowitz (Academic Press, New York, 1988), Vol. 12, p. 335.

[5] M. Ben Amar, P. Pelcé, and P. Tabeling, Growth and Form: Nonlinear Aspects (Plenum Press, New York, 1991).

[6] J. Nittman and H. E. Stanley, Nature (London) 321, 663 (1986).

17) H. Brune, Ch. Romainczyk, H. Roder, and K. Kern, Nature (London) 369, 469 (1994

[8] Th. Michely, M. Hohage, M. Bott, and G. Comsa. Phys. Rev. Lett. 71, 3943 (1993).

191 H. Röder, H. Brune, J.P. Bucher, and K. Kern, Surf. Sci. 298, 121 (1993); H. Röder, E. Hahn, H. Brune, J. P. Bucher, and K. Kern, Nature (London) 366, 141 (1993).

[10] A similar setup based on our original design has recently been described in S. Horch, P. Zeppenfeld, R. David, and G. Comsa, Rev. Sci. Instrum. 65, 3204 (1994).

[11] Ch. Romainczyk, Ph.D. thesis No. 1289, EPF Lausanne (1994).

[12] Similar branch widths have been observed for Au fractals on Ru(0001) at 300 K. R. Q. Hwang, J. Schröder, C. Günther, and R. J. Behm, Phys. Rev. Lett. 67, 3297 (1991).

[13] H. Brune, H. Röder, C, Boragno, and K. Kern. Phys. Rev. Lett. 73, 1955 (1994).

[14] A. Pimpinelli, J. Villain, and D. Wolf, J. Phys. (Paris) 3, 447 (1993)

[15] Pimpinelli et al. considered the nucleation probability for a distribution of edge aroms rather than for one adatom resulting in the instability criterion $L_{c} \approx\left(D_{c} / l\right)^{1 / 3}$. This is, however, justified only for a flux $I$ much higher than applied in the present experiment.

[16] M. C. Bartelr and J. W. Evans, Surf. Sci. Lett. 314, L829 (1994).

[17] J. Wintterlin, J. Wiechers, H. Brune, T. Gritsch, H. Höfer, and R. J. Behm, Phys. Rev. Lett. 62, 59 (1989).

[18] H. Brune, K. Bromann, H. Röder, and K. Kern (to be published).

[19] Z. Zhang, X. Chen, and M. Lagally, Phys. Rev. Lett. 73, 1829 (1994).

120) E. Ganz, S. K. Theiss, I. Hwang, and J. Golovchenko, Phys. Rev. Lett. 68, 1567 (1992).

[21] L. D. Roelofs, J. I. Martin, and R. Sheth, Surf. Sci. 250, 17 (1991).

[22] S. C. Wang and G. Ehrlich, Phys. Rev. Lett. 70, 41 (1993).

[23] S. C. Wang and G. Ehrlich, Phys. Rev. Lett. 71, 4174 (1993). 